Journal of Engineering and Applied Sciences 15 (1): 1-3, 2020

ISSN: 1816-949X

(C) Medwell Journals, 2020

\title{
Developing an Arabic Handwritten Recognition System by Means of Artificial Neural Network
}

\author{
Ali Mohsin and Mohammed Sadoon \\ Department of Computer Engineering, College of Engineering, \\ University of Baghdad, Baghdad, Iraq
}

\begin{abstract}
The matter of handwritten text recognition is as yet a major challenge to mainstream researchers. A few ways deal with this challenge have been endeavored in the most recent years, for the most part concentrating on the English pre-printed or handwritten characters space. Consequently, the need to effort a research concerning to Arabic texts handwritten recognition. The Arabic handwriting presents unique technical difficulties because it is cursive, right to left in writing and the letters convert its shapes and structures when it is putted at initial, middle, isolation or at the end of words. In this study, the Arabic text recognition is developed and designed to recognize image of Arabic text/characters. The proposed model gets a single line of Arabic text, which convert and segments into words and then segments into letters. A multilayer feed forward neural network is trained to recognize these segments as characters. The final results indicate and clarify that the proposed system perform an effective accuracy of recognition rated up to $83 \%$ for Arabic text.
\end{abstract}

Key words: Arabic text, isolation, model, recognize, effective accuracy, challenge

\section{INTRODUCTION}

Many applications depend on automatic text recognition technique such as searching for words in extensive volumes of papers, and appropriate editing of previously printed documents, automatic arranging of postal mail, cheque verification. This research can be divided into three main steps. The first step was the pre-processing stage, the second was feature extraction, and the third is classification (Khorsheed, 2000). The pre-processing steps are: scanning, binarization, noise removal, thinning, base line detection and segmentation. The feature extraction summarizes high level information concerning individual patterns to simplify recognition task. This stage tries to extract the important feature vector of Arabic handwritten characters. This vector then will be used in the classifier to recognize the pattern using the feature vector. The feature vector is feeded to neural network in classification stage.

\section{MATERIALS AND METHODS}

Images acquisition: This is the first stage in the proposed system. Process starts with the collection of images from data entry form documents that had been filled by handwritten text. This process is done through scanning process and scanned in digital form by camera of mobile (Fig. 1).

Noise reduction: The noise imposed in this study is salt and pepper noise because it is commonly. Salt-and-pepper "noise is sometimes called impulsive noise or spike noise. An image containing salt-and-pepper noises will have bright pixels in dark regions and dark pixels in bright regions. This type of noise can be caused by bit error in transmission, Dead pixel in an LCD monitor etc. It can be mostly eliminated by using median filter (Fig. 2).

Median filter is preserving edges in an images while decreasing random noise. The templates of size $3 \times 3$ is applied to each pixel (Fig. 3). The values within this template are sorted and the center of the sorted list is used to replace the template central pixel, several filters were examined however, a $3 \times 3$ median filter was selected because it gave us the preferable result (Aouadi and Echi, 2016).

Color to gray scale level: The entered scanned form images are transformed to grey scale using the typical conversion in Eq. 1 (Fig. 4):

$$
\text { Grey level }=0.299 * \mathrm{R}+0.587 * \mathrm{G}+0.114 * \mathrm{~B}
$$

where, R, G and B symbolize the colors red, green and blue respectively, with weights from 0-255.

Image binarization: Binarization refers to the conversion of the gray scale images to a binary images (Fig. 5). In our methodology, binarization is a function that turns the values of the pixels weights into two values. These values are black (weight 0 ) and white (weight 255). The Binarization operation is useful in this project where the need to clarify the main objects in the data entry form image such as characters or word (Ali et al., 2015).

The Threshold value (T) should be entered which represents the weight value where all weight values in all

Corresponding Author: Ali Mohsin, Department of Computer Engineering, College of Engineering, University of Baghdad, Baghdad, Iraq 


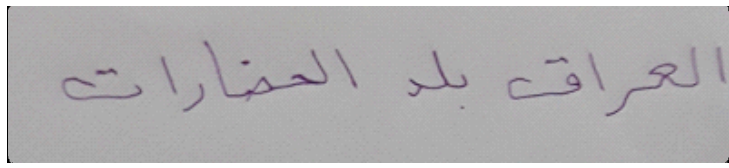

Fig. 1: Handwritten image

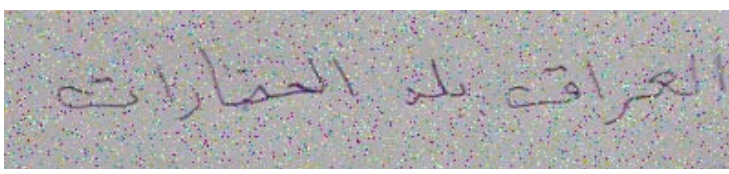

Fig. 2: Image with salt pepper noise

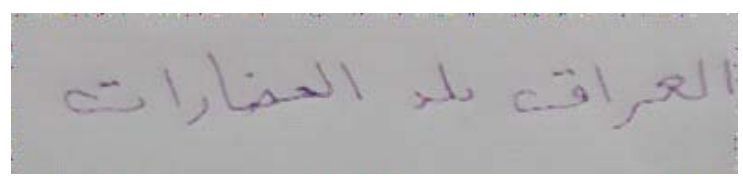

Fig. 3: Image after applying $3 \times 3$ median filter

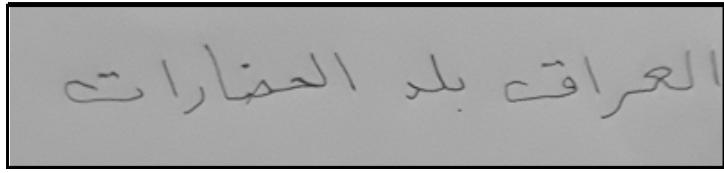

Fig. 4: Image in gray scale

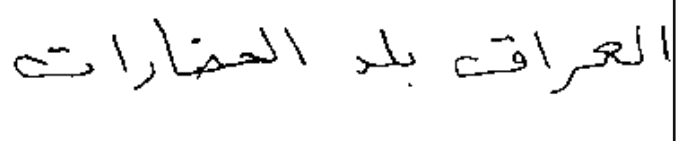

Fig. 5: Binary image

\section{العرافته بلد الميماراته}

Fig. 6: After applying dilation

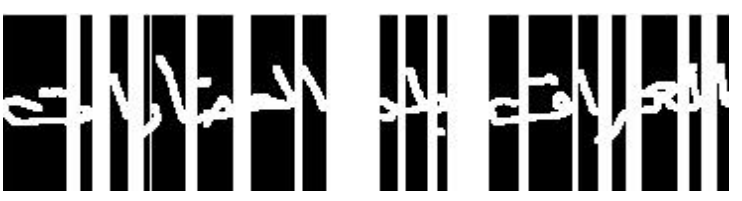

Fig. 7 after segmentation

pixels will be separated to either black if above the threshold value or white if less than the threshold value. The binarization applied on data entry form using a threshold value 180 as follow:

- If pixel value $>=180$ then pixel value $=255$ (white)

- $\quad$ Else
- $\quad$ If pixel value $<180$ then pixel value $=0$ (black)

If the images suffering from an extreme case of nonuniform illumination the threshold of binarization should be adaptive, so that, the foreground pixels (representing script characters) are darker than the background pixels (the white paper).

The algorithm called Bradley's method. This method uses the large neighborhood mean filters. If the input image pixel is more than a certain percentage greater than the mean filter, then it is set to white. The dimension of mean filter in this method is not fixed in this research a square neighborhood is used that is about $1 / 8$ of the smallest image dimension.

Thinning: It is a process which increases or thickens objects in the binary images. After applying binarization, another problem is appeared which is the erosion or broken characters. the proposed methodology solves this problem based on using morphology dilation to reconstruction the missing pixels in the character according to dilate proposed structuring element array with the handwritten character image's array to fill the gap in the character (Fig. 6).

The dilation operation is executed by laying the structuring element on the picture and sliding it across the picture in a way like to convolution. which explained as follow:

- If the origin of the structuring element coincides with a " 0 " in the image, there is no change; move to the next pixel

- If the origin of the structuring element coincides with a " 1 " in the image, execute the OR logic process on each pixels within the structuring element

- A $3 \times 3$ square structuring element was selected because it gave us the preferable result (Umbaugh, 2010)

\section{RESULTS AND DISCUSSION}

Segmentation: The goal of a character segmentation algorithm is to partition a word image into regions, each containing an isolated complete character. The initial segmentation of characters can make the difference between very good and very poor results for recognition system.

The operation starts by constructing a histogram done by counting the number of black pixels in each column. The beginning of each character is identified by sensing a sudden change in the histogram to minimum peak. The algorithm creates a large number of tiny segments which may not be a letter as a whole, to recover from this error a threshold value is chosen, if a segment is less than the threshold, it is combined with earlier character encountered otherwise if the segment is larger than a threshold value it is set to undergo for a horizontal segmentation (Al-Ma’adeed, 2004) (Fig. 7). 
Feature extraction: The process of transforming the input data into a set of features which can very well represent the input data. Moment can be used to differentiate characters (م-,,$-\varepsilon-$ ) from each other, or other feature extraction techniques. The moment features capture the global shape information. Let the coordinates of a generic black pixel be represented by (r, c). For the 2-D binary images of a segment (may be a complete character), the central moments defined by Jumari and Ali (2002).

Classification: Experimental results are promising, give a recognition rate about $83 \%$ for all characters although, some characters get success rate of $96 \%$ accuracy. these results taken from 15 volunteer each one writes 5 sentences and each of these sentences are about 3-7 word. The classifier used in this study is the feed forward neural network.

\begin{tabular}{|c|c|c|c|}
\hline Characters & $\begin{array}{l}\text { Recognition } \\
\text { accuracy (\%) }\end{array}$ & Character & $\begin{array}{c}\text { Recognition } \\
\text { accuracy (\%) }\end{array}$ \\
\hline 1 & 93 & ض & 70 \\
\hline ب & 81 & $b$ & 87 \\
\hline ت & 66 & ط & 79 \\
\hline$ث$ & 73 & $\varepsilon$ & 69 \\
\hline 飞 & 88 & $\dot{\varepsilon}$ & 89 \\
\hline$\tau$ & 91 & ف & 90 \\
\hline$\dot{\tau}$ & 93 & ق & 66 \\
\hline s & 78 & ك & 81 \\
\hline j & 85 & J & 96 \\
\hline J & 86 & م & 90 \\
\hline j & 78 & ن & 96 \\
\hline س - l - l & 73 & o- & 78 \\
\hline ش & 85 & g & 96 \\
\hline ص & 96 & s & 92 \\
\hline
\end{tabular}

\section{CONCLUSION}

This study presents an approach for extracting features and preprocessing for image text to perform high recognition accuracy of handwritten Arabic text. Many algorithms that used during the preprocessing phase comprehensive binarization and noise removing using median filter and thinning using morphological filter, and tackle some of image suffering from an extreme case of nonuniform illumination by using adaptive binarization technique. It furthermore overcomes many of handwritten characters variations. choosing suitable features for recognizing handwritten Arabic characters will give best recognition accuracies, hence, the feature in this model included seven moment invariant which it is invariant to rotation, size and translation of character. Despite the fact that, there are several challenges with some characters, however, the overall recognition rate is good particularly when compared to other handwritten Arabic character's systems. After checking the recognition accuracy of every characters it is found that the recognition rate is between $96 \%$ for the easiest recognized letters such as (ص , ن ,و ) and 66\% for the hardest recognized letters like ( ) ( ), possibly this is because those letters include loops and also, they can have various characteristics from one to other writing style. letters with dots furthermore tend to have low recognition accuracies because the differences in drawing the dots (dots may be linked to the main body of the letter or even can be removed in other writing styles) give inaccuracies.

\section{REFERENCES}

Al-Ma'adeed, S.A.S., 2004. Recognition of off-line handwritten Arabic words. Ph.D. Thesis, University of Nottingham, Nottingham, England.

Ali, O.B., A. Shaout and M. Elhafiz, 2015. Two stage classifier for Arabic handwritten character recognition. Int. J. Adv. Res. Comput. Commun. Eng., 4: 646-650.

Aouadi, N. and A.K. Echi, 2016. Word extraction and recognition in Arabic handwritten text. Intl. J. Comput. Inf. Sci., 12: 17-23.

Jumari, K. and M.A. Ali, 2002. A survey and comparative evaluation of selected off-line Arabic handwritten character recognition systems. J. Teknol., 36: 1-17.

Khorsheed, M.M.S., 2000. Automatic recognition of words in Arabic manuscripts. Ph.D. Thesis, University of Cambridge, Cambridge, England, UK.

Umbaugh, S.E., 2010. Digital Image Processing and Analysis: Human and Computer Vision Applications with CVIPtools. 2nd Edn., CRC Press, Boca Raton, Florida, USA., ISBN: 9781439802052 , Pages: 977. 